\title{
Protrahierte respiratorische Insuffizienz - Epidemiologie und Netzwerk zur Respiratorentwöhnung (Weaning) nach prolongierter Beatmung
}

\author{
Protracted Respiratory Insufficiency - Epidemiology and Network on Respiratory Weaning after Prolonged \\ Ventilation
}

Autoren

Institute

\section{B. Schönhofer ${ }^{1}$, M. Pfeifer ${ }^{2}$, D. Köhler ${ }^{3}$}

Abteilung Pneumologie und Intern. Intensivmedizin, Klinikum Region Hannover

Zentrum für Pneumologie, Krankenhaus Donaustauf

Fachkrankenhaus Kloster Grafschaft, Schmallenberg eingereicht 30.6. 2010 akzeptiert nach Revision 30. 6. 2010

\section{Bibliografie}

DOI http://dx.doi.org/ 10.1055/s-0030-1255624 Pneumologie 2010; 64: 595-599 @ Georg Thieme Verlag KG Stuttgart · New York ISSN 0934-8387

\section{Korrespondenzadresse}

Prof. Dr. med.

\section{Bernd Schönhofer}

Klinik für Pneumologie und internistische Intensivmedizin Krankenhaus OststadtHeidehaus Klinikum Region Hannover $\mathrm{GmbH}$

Podbielskistraße 360 30659 Hannover Bernd.Schönhofer@t-online.de

\section{Zusammenfassung \\ $\nabla$}

Die maschinelle Beatmung polymorbider, alter und pulmonal vorgeschädigter Patienten nimmt zu. Damit steigt auch die Prävalenz des schwierigen oder verzögerten Weanings von Langzeitbeatmung. Spezialisierte Weaningzentren übernehmen schwer entwöhnbare Patienten von Intensivstationen, wo sie langfristig Ressourcen binden. In einer landesweiten Umfrage wurde für das Jahr 2006 eine Bestandsaufnahme des pneumologischen Weanings in Deutschland durchgeführt. In 38 Zentren wurden 2718 Weaningpatienten betreut. Drei Viertel der Patienten wurden zum Weaning aus externen Krankenhäusern in ein Zentrum übernommen. Es ließen sich 66,3\% der Patienten erfolgreich vom Respirator entwöhnen, wobei 31,9\% der Patienten im Anschluss an den Weaningprozess mit Heimbeatmung versorgt wurden. Die Krankenhaussterblichkeit lag bei 20,8\%. Unter der Schirmherrschaft der Deutschen Gesellschaft für Pneumologie und Beatmungsmedizin (DGP) wurde „WeanNet“ das Kompetenznetzwerk pneumologischer Weaningzentren - gegründet. Das wesentliche Ziel von WeanNet ist die Verbesserung der Zusammenarbeit der spezialisierten Weaningzentren und die Qualitätssicherung. Wichtige Instrumente innerhalb von WeanNet sind das Patientenregister und die Akkreditierung der Zentren. In der Entwicklungsphase des Registers kooperierte die WeanNet eng mit dem Institut für Lungenforschung (ILF). Den Betrieb der Datenbank und die Benutzerverwaltung hat das ILF übernommen. Weniger als ein Jahr nach dem Start des Registers haben sich bereits 70 Weaningeinheiten mit ca. 3000 Patienten eingeschrieben. Die Beteiligung am Weaning-Register und die Akkreditierung bilden in Zukunft eine wesentliche Grundlage für die Qualitätssicherung der Weaningzentren.

\section{Abstract \\ $\nabla$}

The prevalence of difficult or prolonged weaning from the ventilator is increasing due to a growing number of multi-morbid, elderly and pulmonary deficient patients being mechanically ventilated. Intensive care units (ICU) tend to refer difficult to wean patients to specialised weaning facilities. A survey of 38 centres - performed in 2006 - included a total number of 2718 patients with difficult or prolonged weaning. Almost three quarters of the patients were transferred to a weaning centre from an external ICU. The weaning success rate was $66.3 \%$. After weaning in $31.9 \%$ of the patients, home mechanical ventilation was started. The overall hospital mortality rate was $20.8 \%$. Recently the task force „WeanNet“ - a network of weaning units - was founded under the auspices of the German Thoracic Society. The main aim of WeanNet is to improve cooperation among the weaning centres and the quality of patient management. Important tools of WeanNet are (i) the register of weaning patients and (ii) accreditation of the weaning centres. To develop the register an intensive cooperation between the task force and the Institute for Lung Research (ILF) was necessary. The finished register is now logistically run by ILF. In less than 1 year after the official start, already 70 weaning units with ca. 3000 patients are registered. In future „WeanNet“, in particular in terms of the register and the accreditation, will stand for the quality of weaning centres in Germany. 


\section{Hintergrund}

$\nabla$

In der Intensivmedizin kommt der maschinellen Beatmung eine zentrale Bedeutung zu. Patienten werden sowohl elektiv, z.B. während chirurgischer Eingriffe, als auch zur Überbrückung vital bedrohlicher Situationen maschinell beatmet.

Seit Jahren steigt die Anzahl der intensivmedizinisch zu versorgenden Patienten, die nicht zügig vom Respirator entwöhnt werden können. Cox et al. zeigten eindrucksvoll, dass die Prävalenz des Weaningversagens im Beobachtungszeitraum von 1993 bis 2002 um ca. 40\% gestiegen ist [1]. Der Anstieg der Patienten, die schwierig vom Respirator zu entwöhnen sind, ist multifaktoriell verursacht [2], resultiert aber wesentlich aus der zunehmenden Zahl polymorbider und alter Patienten bei gleichzeitig verbesserter intensivmedizinischer Versorgung.

Obwohl es bei nur etwa 10\% der beatmeten Patienten zur Langzeitbeatmung (LZB) kommt, verbrauchen diese ca. 50\% der Ressourcen der Intensivstation [3]. Für nicht auf Beatmung spezialisierte Intensivstationen stellen polymorbide Patienten, die nur mit großem Aufwand vom Respirator entwöhnt oder sogar langzeitbeatmet werden, häufig eine Überforderung dar.

\section{Historie}

$\nabla$

Lange Zeit existierte in der deutschen Gesellschaft für Pneumologie (DGP) kein offizielles Mandat für den Bereich Intensivmedizin. Angelehnt an die internationale Entwicklung der pneumologisch orientierten Intensivmedizin in der European Respiratory Society (ERS) und der American Thoracic Society (ATS) wurde dann schließlich im Jahr 2001 innerhalb der DGP die Sektion Intensivmedizin gegründet.

Eine zuvor im Jahre 1999 durchgeführte Umfrage ergab, dass die Pneumologie in der internistischen Intensivmedizin erheblich unterrepräsentiert ist, und zwar sowohl in kleineren Krankenhäusern als auch in großen Schwerpunktkliniken, insbesondere aber auch an den Universitätskliniken.

Für die anfangs umstrittene, im weiteren Verlauf aber mit großer Akzeptanz bestätigte Namensänderung unserer Gesellschaft in „Deutsche Gesellschaft für Pneumologie und Beatmungsmedizin“ auf der Jahrestagung 2005 in Berlin war im Wesentlichen der damalige Präsident Prof. Dr. Dieter Köhler verantwortlich. Die Namensänderung war ein wichtiger Schritt zur Integration der Intensivmedizin in die Pneumologie. Analog hierzu wurde bei der Jahrestagung der DGP 2006 in Nürnberg durch Mitgliederentscheidung der bisherige Name der Sektion „Intensivmedizin“ in „Intensiv- und Beatmungsmedizin“ geändert. Durch diese Namensänderungen wird der interessierten Öffentlichkeit vermittelt, dass die Beatmungsmedizin innerhalb der pneumologischen Intensivmedizin einen herausragenden Stellenwert einnimmt.

Wichtigstes Ziel der DGP-Sektion Intensivmedizin ist es daher gewesen, die Pneumologie in der internistischen Intensivmedizin zu verankern und interessierten Pneumologen den Zugang in die internistische Intensivmedizin zu ermöglichen. Die Sektion Intensivmedizin ist aus diesem Grund bestrebt, die Intensivund Beatmungsmedizin als unverzichtbares Element der pneumologischen Weiterbildung zu etablieren.

Wir verbinden damit aber auch die Einladung an assoziierte Berufsgruppen, wie vor allem Atmungstherapeuten, Physiotherapeuten und Pflegekräfte, in der Sektion Intensiv- und Beatmungsmedizin aktiv mitzuarbeiten.
Im September 2006 wurde das 1. Curriculum Beatmungsmedizin der DGP erfolgreich ausgerichtet. An interaktiven Workshops mit vier Modulen zu aktuellen Themen der invasiven und nichtinvasiven Beatmung nahmen Ärztinnen und Ärzte vorwiegend aus pneumologischen Abteilungen teil. Die Resonanz der Teilnehmer war sehr positiv, sodass die Veranstaltung seitdem jährlich an verschiedenen Orten innerhalb Deutschlands durchgeführt wird.

\section{WeanNet - das Netzwerk der Weaningzentren $\nabla$}

In den USA wurden bereits vor ca. 30 Jahren spezialisierte Weaning-Einheiten zur Entlastung der Intensivstationen, zur Verbesserung der Prognose und zur Kostenreduktion eingerichtet $[4,5]$. Die wachsende Bedeutung der Beatmungsmedizin wird auch durch die Versorgungsrealität unterstrichen: In den vergangenen 20 Jahren hat sich auch in Deutschland eine kontinuierlich zunehmende Anzahl pneumologischer Kliniken und Abteilungen auf die schwierige Entwöhnung vom Respirator spezialisiert.

\section{Strategien}

Die Erschöpfung der Atmungsmuskulatur ist die wesentliche Ursache für die erfolglose Entwöhnung vom Respirator. In spezialisierten Entwöhnungseinheiten gelingt die Respiratorentwöhnung mit Hilfe einer therapeutischen Gesamtstrategie relativ häufig [6]. Es ist wichtig, bereits zum Zeitpunkt der Intubation und in der frühen Beatmungsphase wichtige Prinzipien der Respiratorentwöhnung zu beachten, um spätere Komplikationen und eine LZB möglichst zu verhindern.

\section{AG Pneumologische Weaningzentren}

Mit der Aufgabe, die Struktur-, Prozess- und Ergebnisqualität der pneumologischen Weaningzentren zu sichern, beauftragte die DGP 2006 eine Arbeitsgruppe „Pneumologische Weaningzentren“. Mitglieder der Arbeitsgruppe waren: U. Achtzehn, Chemnitz; T. Barchfeld und D. Köhler, Grafschaft; J. Geiseler, Gauting; F. Heinemann, Donaustauf; F. Herth, Heidelberg; C. Kelbel, Dortmund; B. Schönhofer, Hannover (Federführung); B. Schucher, Großhansdorf und M. Westhoff, Hemer.

\section{Epidemiologie}

Als Grundlage für ein zukünftiges Akkreditierungsverfahren sowie ein Netzwerk für Weaningzentren führte die Arbeitsgruppe „Pneumologische Weaningzentren“ eine Erhebung zur aktuellen Struktur und Outcomedaten der pneumologischen Weaningzentren durch [6]. Der erfasste Zeitraum reichte von Januar bis Dezember 2006. An der Erhebung mit 10 Kategorien zu Daten des Zentrums, Patienten, Weaningstrategien und Outcomes nahmen 38 Zentren teil, in denen 2718 Weaningpatienten betreut wurden. Drei Viertel der Patienten wurden zum Weaning aus externen Krankenhäusern in ein Zentrum übernommen.

Entsprechend der Kategorien zum Weaning, die die internationale Konsensus-Konferenz formuliert hat [7], gehören 27,7\% der Patienten zur Gruppe „Schwieriges Weaning“ und 72,3\% in die Gruppe „Prolongiertes Weaning“.

Es ließen sich 66,3\% der Patienten erfolgreich vom Respirator entwöhnen, wobei 31,9\% der Patienten im Anschluss an den Weaningprozess mit Heimbeatmung versorgt wurden. Die Krankenhausmortalität betrug im Mittel 20,8\%. Bezüglich der Patientenzahlen, Organisation und Weaningstrategien gibt es z.T. deutliche Unterschiede zwischen den Zentren. Aufgrund dieser Erhebung wurde deutlich, dass die pneumologischen Zentren einen 


\section{Tab. 1 Aufgaben von WeanNet.}

\section{Erarbeitung von Weaning-Standards}

Harmonisierung der Übernahme- und Überleitungsschnittstellen; Erstellung von Fragebögen zur einheitlichen Charakterisierung de Patienten vor der Übernahme ins Weaningzentrum

Erstellung einheitlicher Erfassungskriterien bzgl. Entlassung und Überleitung in weiterbehandelnde Institutionen

Erstellung einer einheitlichen und Leitlinien-konformen Zusammenfassung der wichtigsten Definitionen, deren Bedeutung und Prozesse zum Thema Weaning

Entwicklung einer einheitlichen Datenerfassung bzgl. Aufnahme- und Entlassmanagement, Patientencharakteristika, Prozess und Outcome Entwicklung von zentrumsübergreifenden Definitionen von Qualitätszielen

Datenaustausch und -vergleich der Zentren untereinander

Entwicklung eines Konsens und Stratifizierung für Weaningstrategien

Erarbeitung eines Konsenses bzgl. unterstützender Berufsgruppen

bzw. deren Ausbildungsstandards

Unterstützung bei der Entwicklung von typischen Informations-

elementen und Schulungsprogrammen

wesentlichen Beitrag in der Versorgung beatmeter Patienten und zur Entlastung nicht spezialisierter Intensivstationen leisten.

\section{"WeanNet" - das Kompetenznetzwerk pneumologischer Weaningzentren}

Auf der Basis der besagten Erhebung und in enger Zusammenarbeit mit der AG Pneumologische Weaningzentren gründete die DGP mit „WeanNet“ ein Kompetenznetzwerk pneumologischer Weaningzentren, das die Zusammenarbeit der spezialisierten Weaningzentren intensivieren und Qualitätssicherung erreichen soll. Die wesentlichen Aufgabengebiete sind in $\bullet$ Tab. 1 aufgeführt.

Gemeinsam entwickelten die $A G$ „Kompetenznetzwerk pneumologischer Weaningzentren “ und das Institut für Lungenforschung (ILF; Direktorin: Frau Dr. Nina Hämäläinen) ein zentrales Patientenregister zur Erfassung von Weaningpatienten und ein Akkreditierungsverfahren für Weaningzentren. Weitere Details der in - Abb. 1 dargestellten Synergien von Register und Akkreditierungsverfahren werden im Folgenden erläutert.

\section{Patientenregister}

Das Weaning-Register erfasst Patienten, die entsprechend dem internationalen Konsensus [7] langzeitbeatmet - d.h. länger als 7 Tage - sind und zur Respiratorentwöhnung in ein spezielles Weaningzentrum verlegt wurden. Das Register umfasst anamnestische und diagnostische Daten, Angaben zu Dauer und Verlauf der Beatmung, zum Prozedere der Entwöhnung, Daten zu aufgetretenen Komplikationen und zum Outcome der Patienten. Es wurde auf einen möglichst schlanken Datensatz, der auf medizinisch zentrale und klinisch relevante Informationen beschränkt ist, geachtet.

Für die Programmierung, die Pflege und den Betrieb der Datenbank ist das ILF verantwortlich. Nach Abschluss der Testphase mit den in der AG vertretenen Weaningzentren steht das internetbasierte Weaning-Register inzwischen allen Weaningeinheiten mit Zugangsberechtigung bundesweit zur Verfügung. Die Anwender werden bei der Dateneingabe durch interaktive Hilfen unterstützt. Um die Vorgaben des Datenschutzes der Patienten zu erfüllen, werden die Angaben pseudonymisiert. Für die Patientenaufklärung und Einwilligung stellt das ILF die notwendigen Unterlagen zur Verfügung.

Das Angebot des Registers wurde inzwischen von den pneumologischen Weaningzentren erfolgreich angenommen [8]. Innerhalb eines Jahres nach dem Start des Registers haben sich bereits 70 Weaningeinheiten mit insgesamt ca. 3000 Patienten eingeschrieben.

\section{Akkreditierung der Weaningzentren}

Ziel der Akkreditierung ist die Verbesserung der medizinischen Behandlungsabläufe und eine externe Qualitätssicherung. Getragen wird das Projekt von der Erkenntnis, dass Weaning von langzeitbeatmeten Patienten erfolgreich sein kann, wenn neben der medizinischen Kompetenz eine spezifische Infra- und Organisationsstruktur, d. h. Struktur- und Prozessqualität vorhanden ist. Die Voraussetzungen zur Akkreditierung eines Weaningzentrums und die konkrete Planung des Akkreditierungsprozesses wurden von der AG WeanNet erarbeitet. Der Kriterienkatalog ist über die Webseite der DGP abrufbar. In $\bullet$ Tab. 2 sind die wesentlichen Themengebiete aufgeführt.

Die Dokumentation von mindestens 40 Patienten mit schwierigem Weaning innerhalb eines Jahres im Weaning-Register ist die entscheidende Voraussetzung, um die Akkreditierung beantragen zu können. Auch nach dem erfolgreichen Erwerb der

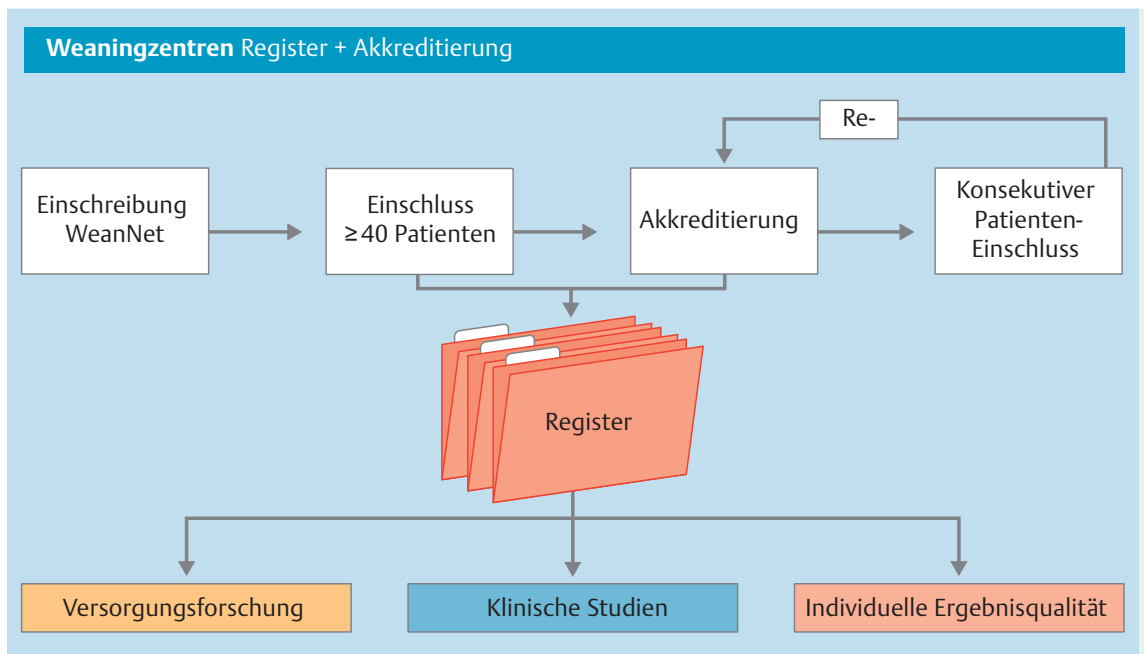

Abb. 1 Algorithmus zur Interaktion von Patientenregister und Akkreditierungsverfahren der Weaningzentren. 
Tab. 2 Kriterienkatalog zur Akkreditierung von Weaningzentren.
1. Generelle Anforderungen an das Weaningzentrum
- Anforderungen an die Qualifikation des ärztlichen Leiters
2. Integriertes Behandlungskonzept
- internistische Intensivstation
- Weaningeinheit
- Heimbeatmungsstation
3. Technische Ausstattung
- Respiratoren
- Interfaces
- sonstige Ausstattung
- Spezialausstattung für Risiko-Patientengruppen
4. Personalausstattung
- ärztliches Personal
- Pflegepersonal
- Physiotherapie/Atemtherapie
- Fortbildung
5. Prozessqualität
- Anzahl Weaningpatienten
- Weaningstrategien
- Patientenübernahme/Schulung/Überleitung
Anhänge überarbeiten, Übernahmefragebogen entwickeln
- Arztbriefe
- Weaning am Lebensende
6. Ergebnisqualität
- Teilnahme am Weaningregister
- Qualitätsmanagement

Akkreditierung ist die weitere Teilnahme des Weaningzentrums am Register verpflichtend.

Im Herbst 2009 fand ein Lehrgang für Weaning-Experten zur Qualifikation zu Auditoren statt. Schließlich wurden im Rahmen einer vorgeschalteten Pilotphase vier Weaningzentren von Auditoren besucht, um die Praktikabilität des Akkreditierungsverfahrens und des Kriterienkatalogs während des Audits zu überprüfen. Basierend auf den Erkenntnissen wurden Änderungen am ursprünglich vorgesehenen Ablauf des Audits und am Formularwesen der Akkreditierung vorgenommen. Nach Auswertung der Pilotphase wurde das Akkreditierungsverfahren im Mai 2010 abgeschlossen und allgemein zugänglich gemacht. Bisher haben sich 20 Zentren zur Akkreditierung angemeldet. In der 2. Jahreshälfte 2010 finden noch in 10 Weaningzentren Audits statt.

Im Rahmen des Jahreskongresses der DGP vom 17.-20. März 2010 in Hannover wurde von den Mitgliedern des WeanNet die Geschäftsordnung verabschiedet und der 7-köpfige Vorstand gewählt. Sprecher: B. Schönhofer, stellvertretender Sprecher: J. Geiseler; Mitglieder des Beirates sind D. Köhler, M. Pfeifer, H. Bischoff, S. Rosseau, C. Kelbel. Eine sich aus dem Vorstand rekrutierende Kommission wird in Zukunft den Akkreditierungsprozess begleiten und ggf. weiterentwickeln.

\section{Die S2-Leitlinie „Prolongierte Entwöhnung vom Respirator" \\ $\nabla$}

Unter dem Dach der AWMF (Arbeitsgemeinschaft der Wissenschaftlichen Medizinischen Fachgesellschaften e.V.) und der Federführung der DGP wurden in jüngerer Vergangenheit bereits zwei Leitlinien fertiggestellt, die für die prolongierte Respiratorentwöhnung von Bedeutung sind: 1. Nichtinvasive Beatmung als Therapie der akuten respiratorischen Insuffizienz [9]. 2. Nichtinvasive und invasive Beatmung als Therapie der chronisch respiratorischen Insuffizienz [10]. Sowohl die NIV als auch die außerkli-
Tab. 3 Themenübersicht der S2-Leitlinie „Prolongierte Entwöhnung vom Respirator".

\begin{tabular}{|ll|}
\hline AG 1 & Einleitung und Definitionen \\
\hline AG 2 & Pathophysiologie des Weaningversagen \\
\hline AB 3 & Strategien im Weaningprozess \\
\hline AG 4 & Überleitungsmanagement \& Leben nach LZB \\
\hline AG 5 & Erfolgloses Weaning \& Ethik \\
\hline AG 6 & Die Entwöhnungseinheit \\
\hline AG 7 & Methodik \\
\hline
\end{tabular}

nische Beatmung haben im schwierigen Weaning einen festen Stellenwert.

Seit Herbst 2008 wird ebenfalls unter der Moderation der AWMF und Federführung der DGP eine weitere S2-Leitlinie zum Thema „Prolongierte Respiratorentwöhnung“ entwickelt. Beteiligt sind alle wichtigen mit dem Thema der Leitlinie assoziierten wissenschaftlichen Gesellschaften und Institutionen. Diese Leitlinie soll den aktuellen Wissensstand zum Thema „Prolongierte Entwöhnung vom Respirator“ primär auf der Basis publizierter Evidenz, aber auch gestützt auf die Erfahrung von Experten bei noch fehlenden wissenschaftlichen Daten in der Intensivmedizin etablieren und zur weiteren Qualitätssteigerung der Weaningzentren führen. Inzwischen haben im Rahmen dieser S2-Leitlinie bereits zwei Konsensuskonferenzen (im November 2009 und im Juni 2010) stattgefunden. In sieben Arbeitsgruppen werden die Schwerpunktthemen bearbeitet (siehe Tab. 3). Es wurden bisher ca. 70 Empfehlungen erarbeitet. Die Fertigstellung der Leitlinien ist für Mitte 2011 vorgesehen. Die Empfehlungen der S2Leitlinie werden zukünftig auch in den Akkreditierungsprozess für Weaningzentren einfließen.

\section{Ausblick}

$\nabla$

Die Beteiligung am Weaning-Register und die Akkreditierung bilden die Grundlage für die Qualitätssicherung der Weaningzentren. Mittelfristig wird das Weaning-Register auch für Kostenträger und Gesundheitspolitik verlässliche Zahlen zur Versorgungssituation innerhalb der Beatmungsmedizin und Respiratorentwöhnung in Deutschland liefern können.

Mit der Gründung des Kompetenznetzwerks „WeanNet“ besteht die Möglichkeit, verlässliche Daten nicht nur der einzelnen Zentren, sondern auch zur bundesweiten Situation bzgl. Weaning nach Langzeitbeatmung zu liefern. Dies ist die Basis zur Durchführung klinischer Studien und für die Versorgungsforschung. Das Ziel der Versorgungsforschung ist, grundlegendes und anwendungsnahes Wissen über die Praxis der Kranken- und Gesundheitsversorgung zu generieren und der Öffentlichkeit zur Verfügung zu stellen. Aus der Sicht der Versorgungsforschung ergeben sich konkret folgende Themenbereiche für WeanNet: Forschung zum Bedarf der Weaningzentren, zur Versorgungsökonomie und schließlich Versorgungsepidemiologie. Die zur Zeit interdisziplinär erarbeitete S2-Leitlinie „Prolongierte Entwöhnung vom Respirator“ wird zukünftig richtungsweisend sein.

\section{Interessenkonflikte}

$\nabla$

Die Autoren geben an, dass kein Interessenkonflikt besteht. 


\section{Literatur}

1 Cox CE, Carson SS, Holmes GM et al. Increase in tracheostomy for prolonged mechanical ventilation in North Carolina, 1993-2002. Crit Care Med 2004; 32: 2219-2226

2 MacIntyre NR, Epstein SK, Carson S et al. Management of patients requiring prolonged mechanical ventilation: report of a NAMDRC consensus conference. Chest 2005; 128: 3937 - 3954

3 Cohen IL, Booth FV. Cost containment and mechanical ventilation in the United States. New Horiz 1994; 2: 283-290

4 Indihar FJ, Forsberg DP. Experience with a prolonged respiratory care unit. Chest 1982; 81: 189-192

5 Schönhofer B. Schwieriges Weaning nach Langzeitbeatmung. Anästhesiologie und Intensivmedizin 2005; 46: 529-542
6 Schönhofer B, Berndt C, Achtzehn U et al. Weaning in Deutschland - eine Erhebung zur Situation pneumologischer Beatmungszentren. Dtsch Med Wochenschr 2008; 133: 700 - 704

7 Boles JM, Bion J, Connors A et al. Weaning from mechanical ventilation. Eur Respir J 2007; 29: 1033 - 1056

8 Schönhofer B, Teschler H. Start des Weaningregisters - jetzt schon eine Erfolgsgeschichte. Pneumologie 2009; 63: 164-165

9 Schönhofer B, Kuhlen R, Neumann P et al. Nichtinvasive Beatmung als Therapie der akuten respiratorischen Insuffizienz. S3-Leitlinie herausgegeben von der Deutschen Gesellschaft für Pneumologie und Beatmungsmedizin. Pneumologie 2008; 62: 449-479

10 Windisch W, Brambring J, Budweiser S et al. Nichtinvasive und invasive Beatmung als Therapie der chronisch respiratorischen Insuffizienz. S2Leitlinie veröffentlicht von der DGP. Pneumologie 2010; 64: 207-240 